\title{
X-ray tomographic microscopy analysis of the dendrite orientation transition in Al-Zn
}

\author{
Jonathan Friedli ${ }^{1}$, Julie L. Fife ${ }^{1,2}$, Paolo Di Napoli ${ }^{1}$ and Michel \\ Rappaz ${ }^{1}$ \\ ${ }^{1}$ Laboratoire de Simulation des Matériaux, Institut des Matériaux, Ecole Polytechnique \\ Fédérale de Lausanne, Station 12, CH-1015 Lausanne, Switzerland \\ ${ }^{2}$ Laboratory for Synchrotron Radiation, Paul Scherrer Institut, CH-5232 Villigen, Switzerland \\ E-mail: jonathan.friedli@a3.epfl.ch
}

\begin{abstract}
Recently, Gonzales and Rappaz [Met. Mat. Trans. A37:2797, 2006] showed the influence of an increasing zinc content on the growth directions of aluminum dendrites. $\langle 100\rangle$ and $\langle 110\rangle$ dendrites were observed below $25 \mathrm{wt} . \%$ and above $55 \mathrm{wt} . \%$ zinc, respectively, whereas textured seaweeds and $\langle 320\rangle$ dendrites were observed at intermediate compositions. Considering the complexity of these structures, it is necessary to first characterize them in further details and second, to model them using the phase field method. The so-called Dendrite Orientation Transition (DOT) was thus reinvestigated in quenched Bridgman solidification samples. The combination of X-ray tomographic microscopy and electron backscattered diffraction (EBSD) analysis on a whole range of compositions, from 5 to $90 \mathrm{wt} . \% \mathrm{Zn}$, allowed insights with unprecedented details about texture, growth directions and mechanisms of the aforementioned structures. We show that seaweeds rather than dendrites are found at all intermediate compositions. Their growth was confirmed to be constrained within a (100) symmetry plane. However, new findings indicate that the observed macroscopic texture does not necessarily correspond to the actual growth directions of the microstructure. Further, it seems to operate by an alternating growth direction mechanism and could be linked to the competition between the $\langle 100\rangle$ and $\langle 110\rangle$ characters of regular dendrites observed at the limits of the DOT. These characters, as well as 3D seaweeds, are observed in phase-field simulations of equiaxed growth and directional solidification, respectively. This study emphasizes the importance of accurate experimental data to validate numerical models and details the progress that such combinations provide for the understanding of growth mechanisms.
\end{abstract}

\section{Introduction}

Aluminum has a weak anisotropy of the solid-liquid interfacial energy $\gamma_{s \ell}$ (about 1\%) [1] that can easily be perturbed by the addition of an alloying element with a different crystal structure, such as zinc, an hcp element. Recently, a systematic effect of this alloying element has been identified by Gonzales et al [2-4]. These studies showed that an increase in the zinc content in Al-Zn alloys continuously changes the dendrite growth direction from $\langle 100\rangle$ to $\langle 110\rangle$ in a $\{100\}$ plane (Fig. 1). At intermediate compostions, i.e., between 25 and 55 wt.\%, Gonzales et al observed seaweeds at the beginning and end of this dendrite orientation transition (DOT) (areas shaded in grey in Fig. 1) and $\langle 320\rangle$ dendrites around $50 \mathrm{wt. \%}$ Zn. The seaweed microstructures did not exhibit clear trunks and arms but were nevertheless textured. This continuous DOT has a direct 


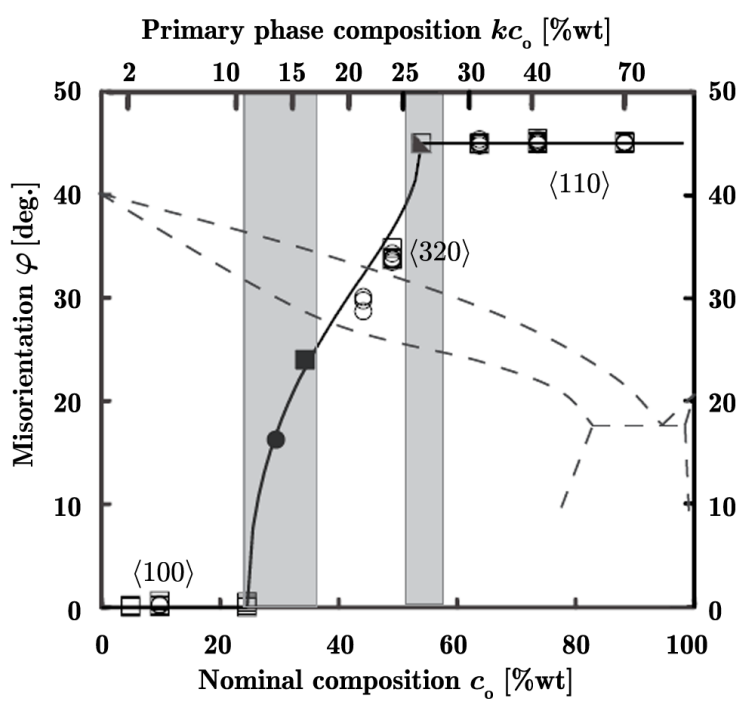

Figure 1. Angle between the $\langle 100\rangle$ direction and the growth direction of $\mathrm{Al}-\mathrm{Zn}$ dendrites as a function of the nominal concentration, $c_{0}$, of $\mathrm{Zn}$. The equilibrium concentration of the first solid, $k c_{0}$, is indicated on the upper scale. Open circles and squares correspond to Bridgman and directional solidification specimens, respectively. The two shaded areas near 25 and 55 wt.\% correspond to concentrations for which textured seaweed structures were observed (filled circles and squares). The high temperature portion of the $\mathrm{Al}-\mathrm{Zn}$ phase diagram is superimposed in dashed lines. [2]
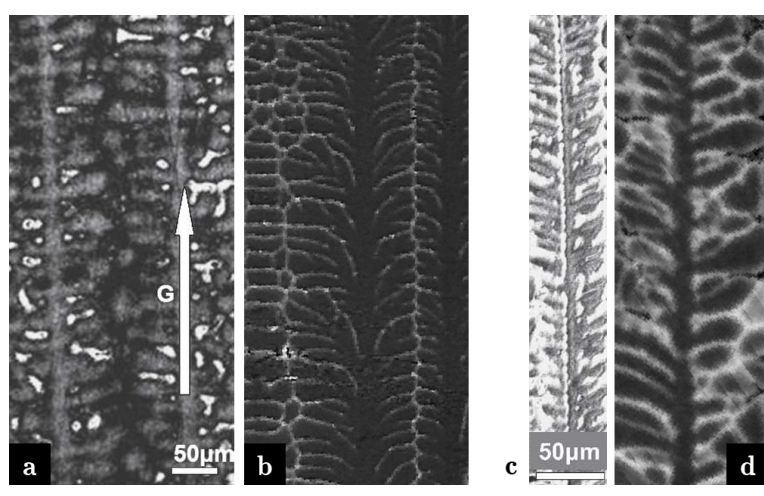

Figure 2. Bridgman solidified Al-10 wt.\% Zn (a,b) and Al-50 wt.\% Zn (c,d) samples. a) Metallographic section in a (100) plane with secondary arms seen at $90^{\circ}$ from the trunk [2]. b) (100) section cutting a trunk in an Xray tomographic microscopy dataset from a quenched sample exhibiting a much more precise view of the secondary arms. c) Reported $\langle 320\rangle$ dendrites [2] showing a trunk and very irregular secondary arms. d) Cut on the X-ray tomographic microscopy stack with an orientation identical to c) through a trunk in a (001) plane showing arms that actually grow out of plane. Note that the magnification is identical for a) and b), and for c) and d), but it does not exactly match between $(a, b)$ and $(\mathrm{c}, \mathrm{d})$.

influence on the texture of directionally solidified alloys [3], and thus has important implications for controlling the mechanical properties of cast alloys.

However, seaweed morphologies were, up to now, almost exclusively analysed in $2 \mathrm{D}$ and found in organic alloys. Furthermore, the existing studies [5-7] never quenched the growing structures and thus, solid-state diffusion during further solidification and cooling smeared the microsegregation pattern formed at an earlier stage. These major difficulties, inherent to the analysis methods used so far for Al-Zn alloys, explain the poor understanding of 3D seaweed formation and orientation selection, and therefore also the lack of a generalized prediction criteria. A study with adapted 3D methods is essential to shed light on such problems, for example by X-ray tomography observations made either in situ on solidifying specimens [8-10] or ex situ on quenched specimens [11].

Therefore, we propose a method enabling the characterization of seaweed and high multiplicity dendritic structures and extend the DOT analysis in Al-Zn alloys. Our experimental procedure encompasses Bridgman solidification and quenching for a wide composition range (from 5 to $90 \mathrm{wt} . \% \mathrm{Zn}$ ), followed by X-ray tomographic microscopy and electron backscattered diffraction (EBSD) measurements. This paper, which presents the experimental results, is complemented by another contribution presenting the 3D phase field simulations. 


\section{Experiments}

\subsection{Bridgman solidification}

The Bridgman setup is a modified version of the vertical tubular furnace presented by Gonzales and Rappaz [2] in their DOT analysis. The modification consisted of a reduction in diameter of the copper water-cooled tube from $10 \mathrm{~mm}$ to $7 \mathrm{~mm}$, allowing for a reduction of the specimen diameter and thus a more efficient quenching. The alloy rods were inserted in quartz or alumina tubes with $4 \mathrm{~mm}$ inner diameter and heated $50^{\circ} \mathrm{C}$ above the liquidus temperature. The samples were then pulled through the furnace at four different velocities $(1,4,10$ and $20 \mathrm{~mm} / \mathrm{min}$ ) in a thermal gradient between 5 and $11 \mathrm{~K} / \mathrm{mm}$ depending on the alloy and tube properties. The temperature gradient was measured by two K-type thermocouples separated by a distance of $10 \mathrm{~mm}$ in the alloy. After $100 \mathrm{~mm}$ of solidification, the specimen was dropped and quenched in water. Gonzales and Rappaz [2] already showed that the pulling velocity, in the range of their Bridgman and directional solidification experiments (i.e. $0.06-3 \mathrm{~mm} / \mathrm{s}$ ), did not influence the DOT. The four velocities in the present case were thus chosen to generate structures fine enough to be representative in the sample volume and coarse enough to be easily distinguishable from the liquid after quenching. The range of cooling rates in the Bridgman furnace varies from 0.08 to $3 \mathrm{~K} / \mathrm{s}$. While the best contrast between the primary solid and the quenched liquid is obtained at low pulling velocities, the cooling rate of the quench $(400-600 \mathrm{~K} / \mathrm{s})$ leads to a fair contrast even in the most unfavorable set of experimental conditions, as confirmed in a previous study of Pompe et al [11]. The metallographic preparation of the quenched samples allowed a precise location of the tip of the structure and thus the location of the tomographic microscopy sample to be extracted. The good phase separation generated by the quench originates from the fact that this method gets rid of backdiffusion during cooling, which greatly simplifies both the subsequent etching procedures and the interpretations of the observed morphologies. This is clearly seen in Fig. 2 where the microstructures reported by Gonzales et al [2] obtained for two alloy compositions are compared with the present ones captured by X-ray tomographic microscopy.

\subsection{X-ray tomographic microscopy}

In order to characterize the complex growth morphologies in 3 dimensions, synchrotron-based X-ray tomographic microscopy experiments were performed at the TOMCAT beamline of the Swiss Light Source (Paul Scherrer Institute, Villigen, Switzerland) [12]. Since the analysis volume accessible through this technique is smaller than the Bridgman sample dimensions, each specimen had to be machined down to the appropriate size at the precise location of the tip of the quenched growth morphology. Diameters from $700 \mu \mathrm{m}$ for highly-alloyed to $2400 \mu \mathrm{m}$ for low-alloyed samples were selected and the specimens then scanned at energies ranging from 20 to $40 \mathrm{keV}$ with pixel sizes from 0.74 to $1.85 \mu \mathrm{m}$. Multiple scans of the height of the sample were taken, aligned, and then merged into one stack to allow for better visualization of the scanned volume.

\section{3. $E B S D$}

The reconstructed tomographic microscopy data stacks were then searched for widely developed secondary arms in a grain reaching up to the quench solidification tip. A transverse cut was then realized with a diamond blade saw at that precise location in the column (e.g., dashed line on Fig. 3b). The sections were then prepared for EBSD mapping.

The SEM micrograph on which the EBSD orientation map is based (e.g., Fig. 3c) was used to retrieve the plane to which it corresponds in the tomographic microscopy dataset. As Fig. 3b shows very well, it is not always exactly perpendicular to the axis of the sample, but this deviation can be accounted for. The orientation map itself was then superimposed on the tomographic microscopy cut (Fig. 3d), the grains identified and their orientation determined. To ensure 
the best possible alignment, when possible, the maps spanned the whole sample. Knowing the misorientation between the EBSD coordinate system and the tomographic microscopy dataset, the orientation of the grains are then rotated from the former to the latter. All planes and directions identified in the tomographic microscopy stack could then be associated with a crystallographic orientation (e.g., Fig. 3e).

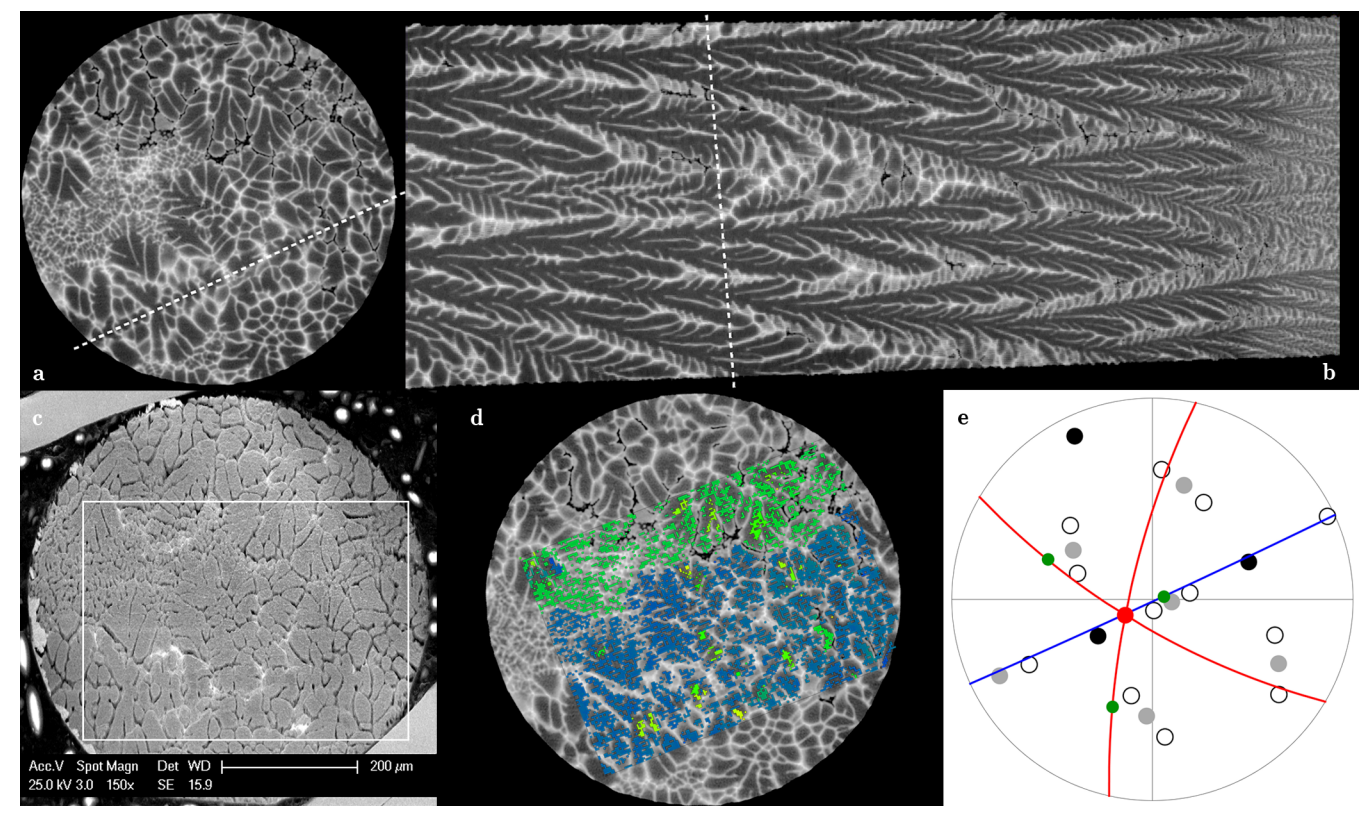

Figure 3. Transverse (a) and longitudinal section (b) of a tomogram of a quenched Al50 wt.\% Zn sample solidified at $4 \mathrm{~mm} / \mathrm{min}$. In the longitudinal section, the thermal gradient is from left to right and the quenched interface can be seen on the right of the image. The dashed lines depict the location of the longitudinal and transverse sections respectively. (c) SEM micrograph of the cut corresponding to (a) through a tomographic microscopy sample, the white rectangle indicates the location of the EBSD map. (d) Same section as (a) with the superimposed EBSD orientation map showing two grains, in green and blue. (e) Typical pole figure in the tomographic microscopy stack coordinate system. The blue and red lines correspond to planes of interest. The red and green dots represent the directions of primary trunks and secondary arms respectively, measured in the 3D reconstruction. The crystallographic orientations obtained from the EBSD map for the corresponding grain are shown by black $\langle 100\rangle$, grey $\langle 110\rangle$ and open $\langle 320\rangle$ circles.

\section{Results and discussion}

As can already be seen in Figs. 2 and 3, the proposed method greatly improves the quality and quantity of information that can be extracted from Bridgman type experiments and enables the clear identification of microstructural features. By quenching, and thus limiting the amount of time the sample remains at high temperatures, the problems of back-diffusion, coalescence and coarsening are avoided and microstructural features are unambiguously identified. Furthermore, as back-diffusion is greatly reduced, the original zinc microsegregation pattern is kept and allows for good contrast during X-ray tomographic microscopy observations.

Two examples of the improvement in interpretation are shown in Fig. 2. In dilute Al10 wt.\% Zn, the secondary arms were believed to grow at an angle of $90^{\circ}$ with respect to the trunk (Fig. 2a). However, Fig. 2b reveals that when cutting exactly through the trunk, 

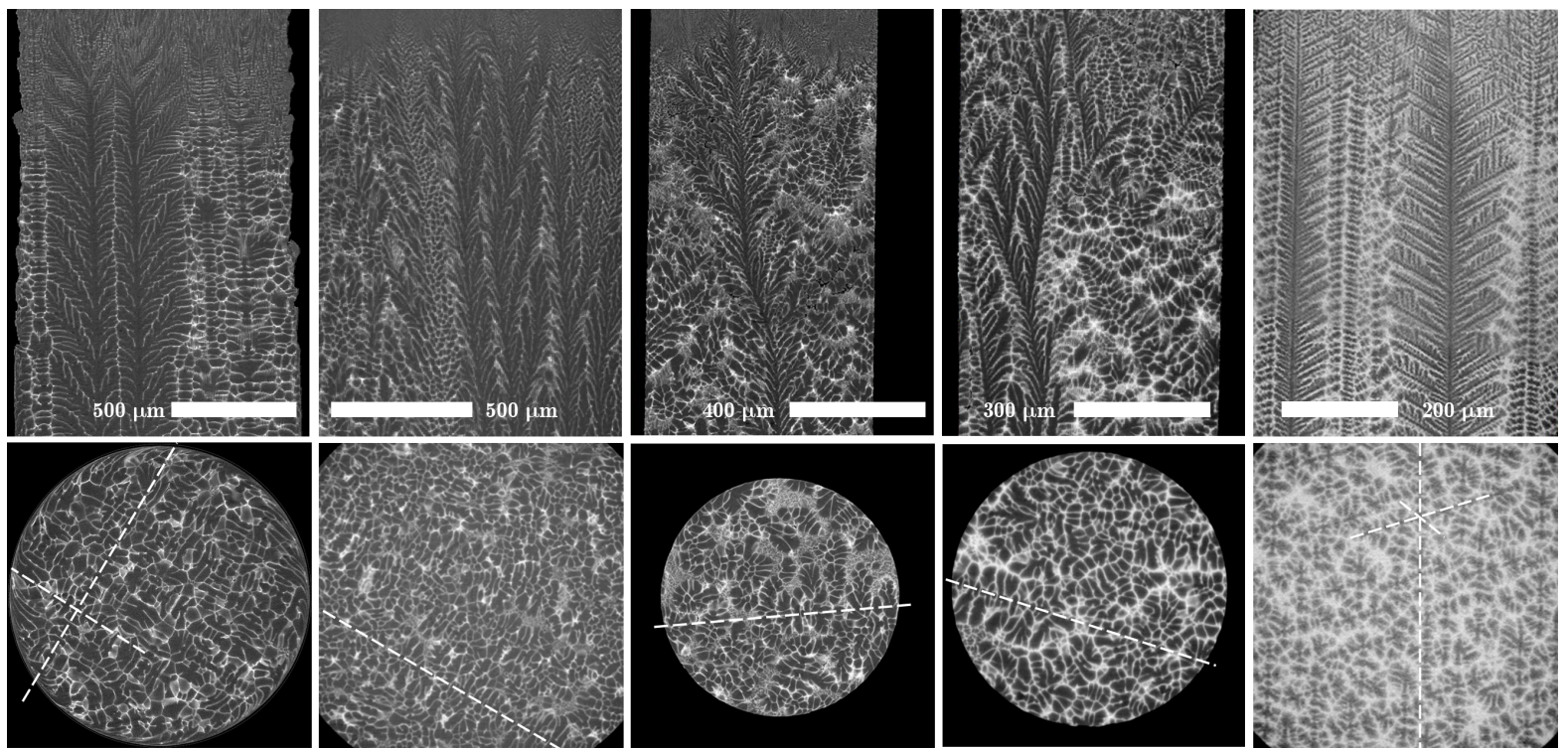

Figure 4. Longitudinal (top) and transverse (bottom) cuts through the X-ray tomography stack. From left to right: Al-20 wt.\% Zn, Al-28 wt.\% Zn, Al-42 wt.\% Zn, Al-55 wt.\% Zn solidified at $10 \mathrm{~mm} / \mathrm{min}$ and Al-82 wt.\% Zn solidified at $20 \mathrm{~mm} / \mathrm{min}$. The trace of the longitudinal section is shown by a dashed white line on the transverse cut. Note that whereas the length scale is the same for longitudinal and transverse sections of each alloy, the magnification varies from one alloy to another.

secondary arms start growing with a smaller angle from the trunk. Moreover, the arms have a tendency to bend and grow almost perpendicularly to the trunks, resulting in a palm-tree morphology. Further analysis of this growth behavior can be found in another paper by the same authors [13]. In the case of the high multiplicity $\langle 320\rangle$ dendrite morphology reported by Gonzales and Rappaz [2] (Fig. 2c), the comparison with the tomographic microscopy data of Fig. 2d shows that the irregularity in the secondary arms comes from out-of-plane growth, suggesting an even more complex growth mechanism than previously expected. These salient features allow for the reexamination of the DOT in Al-Zn.

\subsection{Microstructure analysis}

X-ray tomographic microscopy was performed on Al-Zn alloys from 5 to 90 wt.\% zinc and Fig. 4 shows longitudinal and transverse sections through the stack of samples of various compositions. First, EBSD measurements show an identical angular dependence of the dendrite behavior or seaweed texture as in the DOT for the whole range of Al-Zn alloys, and thereby confirm the results already obtained by Gonzales and Rappaz [2,3]. However, as the last section foretold, the secondary dendrite arms in low-alloyed aluminum and the trunk and arm directions in the intermediate composition range where seaweeds are observed cannot be deduced from the DOT misorientation curve of Gonzales and Rappaz (Fig. 1).

\subsection{Symmetries}

The tomographic microscopy data was scouted for specific symmetry planes, appearing in the transverse cuts (Fig. 4 bottom row). Three types of symmetries have been observed in Al-Zn alloys. Between 5 and 20 wt.\% Zn, trunks with $\langle 100\rangle$ orientation and secondary arms growing in $\{100\}$ planes are observed. In transverse sections those typically appear as crosses with a $90^{\circ}$ angle (Al-20 wt.\% Zn in Fig. 4 bottom row). At zinc concentrations higher than 55 wt.\% , 
dendrites (both trunks and arms) grow along well defined $\langle 110\rangle$ directions. Indeed, in the former studies, the transformation at the eutectic temperature $\left(381{ }^{\circ} \mathrm{C}\right)$ provided some sort of a selfquench [2-4], making the structure clearly appear (e.g., in Al-82 wt.\% Zn Fig. 4 bottom row). This cut also shows the growth advantage, and thus the larger lateral extension, that $\langle 110\rangle$ arms at $60^{\circ}$ from the trunk have over the shorter $\langle 110\rangle$ arms that grow at $90^{\circ}$. In the range of 25 to 55 wt.\% zinc, these symmetries break down and an apparently random structure is observed. Nonetheless, a careful look through the X-ray tomographic microscopy stacks allows one to identify individual symmetry planes in each grain, such as the ones delineated in the bottom row of Fig. 4 for Al-28, 42 and 55 wt.\% Zn.

The EBSD measurements show that in all intermediate compositions investigated, the symmetry plane is a (100) plane, as shown in more details in the example of Al-50 wt.\% Zn (Fig. 3). This finding is in agreement with the previous studies by Gonzales et al since the DOT operates in a (100) plane.

\subsection{Growth mechanisms}

Low and high alloyed Al-Zn alloys exhibit primary dendrite trunks which clearly grow along welldefined $\langle 100\rangle$ and $\langle 110\rangle$ directions, respectively. At intermediate compositions, the situation is more complex since none of these alloys produce a typical dendritic structure, and thus no trunk in the classical sense is present. The three central figures of Fig. 4 (top) shows longitudinal cuts in the symmetry (100) plane of three such alloys: they each exhibit a seaweed-type structure. Instead of well-established trunks, the growth seems to occur with an alternating tip-splitting mechanism with very short arms. The structure is not random however, since certain orientations seems to be preferred in the alternating splitting process. Even if the morphology differs significantly from that of a typical dendrite, one can still identify certain features using the names of more commonly observed structures. We will call a feature a "trunk" if it is aligned within about $20^{\circ}$ of the thermal gradient, and extends over a distance large enough to be clearly identifiable by a number of side branches. Although this definition is imprecise, the trunks in Fig. 4 are nevertheless clear. Any smaller feature which emerges from such a trunk will be called an arm.

To make a meaningful assertion about the trunks, a minimum number showing similar characteristics should be present in a cut. Thus, the sample showing (100) symmetry plane with the largest number of trunks (Al-50 wt.\% Zn, Fig. 3b) was selected and examined in more detail (see Fig. 5). As shown by the solid and dashed white lines in Fig. 5a, two types of trunks were observed: those oriented close to the thermal gradient (dashed lines, e.g., B) show secondary arms on both sides of the trunk in the (100) plane, whereas those further inclined (solid lines, e.g., A1) exhibit arms on only one side in this plane. Note that since the secondary arms are so short and rarely straight, their direction, even taken as the average of the angle with the trunk found at the root, is subject to large errors. The five pole figures shown at the bottom of Fig. 5 correspond to the transverse section of Fig. 3d for this single grain, i.e., the $\langle 100\rangle$ (black dots), $\langle 110\rangle$ (grey dots) and $\langle 320\rangle$ (open circles) are the same. The orientations of trunks and arms in these pole figures are put in relation with the crystallographic orientation of the grain. In Fig. 5e, trunk B grows within a few degrees of the $\langle 320\rangle$ direction whilst trunks A are much more regularly oriented in two directions at $\sim 14^{\circ}$ with respect to $\langle 320\rangle$ (Fig. $5 \mathrm{~b}$ ).

Once examined out of this longitudinal section, the secondary arms emerging from type A trunks are found in two other planes outlined by the two red plane traces in the corresponding stereographic projections. Fig. $5 \mathrm{c}$ and d illustrate this configuration for the trunks A1 and A2. The orientation of these arms in the out-of-plane sections is so ill-defined that it is not possible to identify them with any precise crystallographic direction. The in-plane secondary arms orientation on the other hand, corresponds to the direction of the trunks laying on the other side of the $\langle 320\rangle$ direction, i.e., the in-plane arms of trunk A1 have the orientation of trunk 


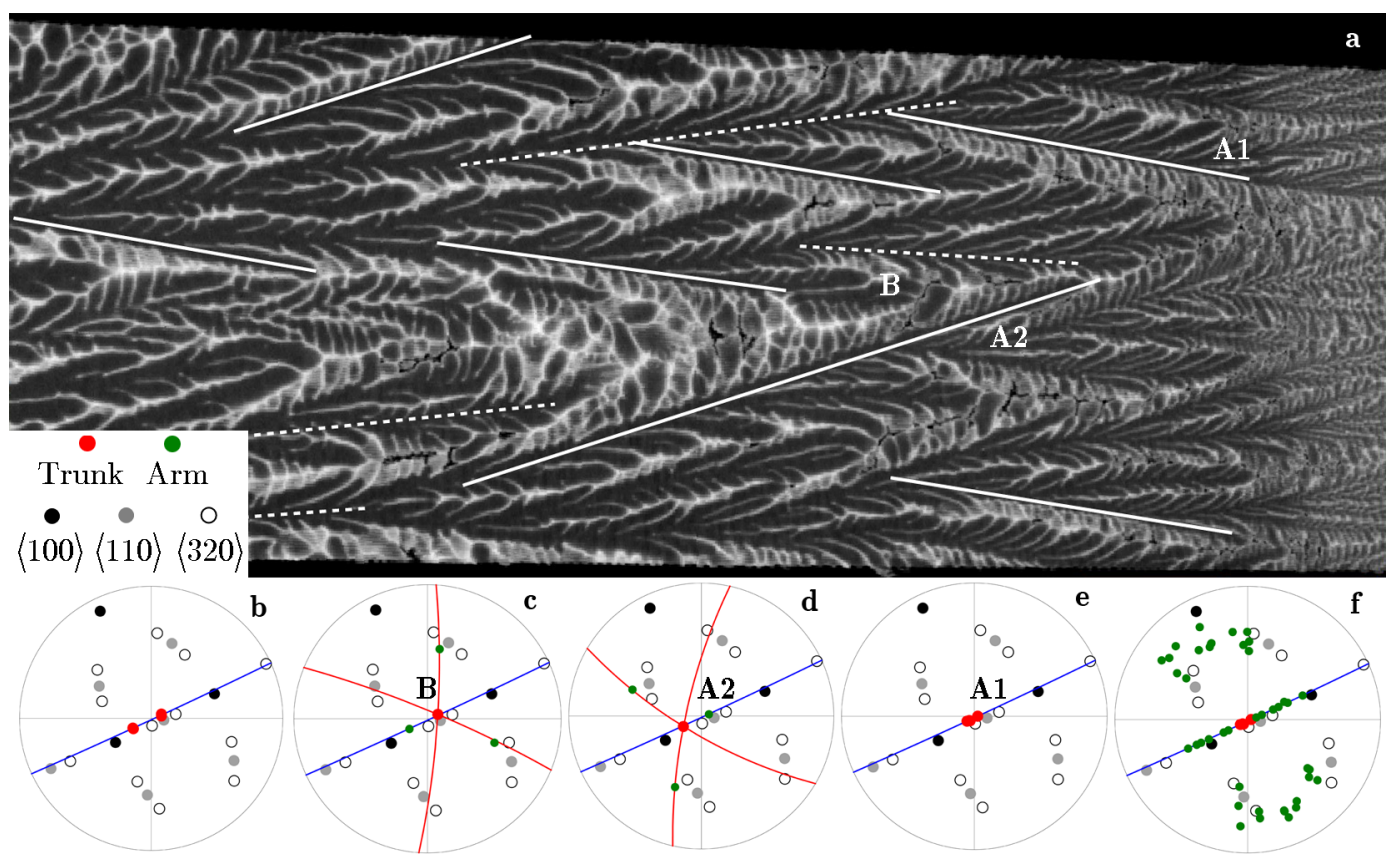

Figure 5. a) (100) symmetry plane in Al-50 wt.\% Zn, showing two types of trunks. Trunks with secondary arms on only one side in the (100) plane, highlighted by solid white lines, and trunks with arms on both sides identified by dashed white lines. b)-f) are the pole figures obtained from the EBSD map in Fig. 3d with the $\langle 320\rangle$ direction being the closest to the vertical, i.e., the thermal gradient direction. The planes and directions found in the X-ray tomography stack are superimposed on the pole figures. The (100) symmetry plane is represented in blue, red and green dots represent trunks and arms, respectively. b) shows the orientation of the A-type trunks. The configuration of the trunk, secondary arms and (red) planes in which the latter lay are shown in c) and d) for trunk A1 and A2, respectively. e) shows the orientation of the B-type trunks, close to the $\langle 320\rangle$ direction. f) shows the directions of the secondary arms associated to the B-type trunks.

A2 and vice-versa. This probably explains why over long distances, a zigzag of the trunks is observed. As such a trunk encounters a perturbation (e.g., the solute field from another trunk or thermal fluctuations), it could switch sides and take the alternate direction. Another example of this phenomenon is shown for Al-42 wt.\% $\mathrm{Zn}$ in the top row of Fig. 4.

The observation of B-type trunks shows a very different configuration. The scatter of the directions in Fig. 5f shows that the secondary arms do not seems to have any preferred growth direction. The potential resulting growth disadvantage could very well explain their short lateral extension compared to the type A configuration.

Although the phase contrast is good, the high variability of features and the feature sizes in contact with the primary solid make the segmentation very tedious and so far impossible to automate. Furthermore, this is a static post-mortem observation of a highly dynamic process and in situ investigations could certainly improve the understanding of this growth morphology.

The observations in Al-50 wt.\% $\mathrm{Zn}$ show that the measurement of the texture of such a sample can be deceptive. Indeed, although the mean growth direction, i.e., the texture, is $\langle 320\rangle$, by far not all actual growth directions correspond to this orientation as evidenced by A-type trunks. 


\section{Conclusion}

A method for investigating growth morphologies in metallic systems, featuring X-ray tomographic microscopy of quenched Bridgman samples, was presented. Focusing on the analysis of complex microstrucutres in Al-Zn alloys, the advantages of this method over other techniques was shown by the clear identification of microstructural features not observable before.

The investigations of the whole hypoeutectic range of Al-Zn alloys, firstly confirmed the texture observations made by Gonzales and Rappaz [2]. At zinc concentrations higher than 55 wt. $\%$, dendrites grow in well defined $\langle 110\rangle$ directions. Below 25 wt. $\% \mathrm{Zn}$, primary trunks grow along $\langle 100\rangle$. Seaweed structures were identified at all compositions between 25 and 55 wt.\% zinc and could, for the first time, be characterized in 3D in a metallic alloy with unprecedented details about texture, growth directions and mechanisms. It was shown that seaweeds are far from random and their growth is constrained within a (100) symmetry plane. However, new findings show that the observed macroscopic texture did not necessarily correspond to the actual growth direction. The growth seems to operate by an alternating growth direction mechanism and could be linked to the competition between $\langle 100\rangle$ and $\langle 110\rangle$ characters, which was simulated using phase field under equiaxed growth conditions $[4,14]$.

Although progresses were made in the understanding of the microstructure formation mechanisms in Al-Zn alloys, some open questions remain. Advancements in the X-ray tomographic microscopy field provide unprecedented spatial and temporal resolution and allow for in situ observations of solidification, which could broaden the spectrum of information available for interpretation. The study of seaweed would certainly most benefit from this technological progress. Recently, TOMCAT has incorporated a laser-based heating system [15] that provides near-isothermal and directional heating capabilities in a broad range of temperatures, which enables in situ observations of growth phenomena in metallic and other materials systems. Finally, a companion paper in this proceedings [14] will show that phase field simulations for alloys exhibiting a complex, but weak, solid-liquid interfacial energy produce hyperbranched dendrites under equiaxed conditions (see also [4]), but much more complex structures, similar to the ones observed here, when such alloys are solidified in a thermal gradient.

\section{Acknowledgments}

The authors gratefully acknowledge the financial support of the Fonds National Suisse pour la recherche scientifique (Grant No 200020-113260 and 121598) as well as the financial and technical support of the Paul Scherrer Institute (Proposal No. 20080240, 20080846, 20090118, 20090795). They would also like to thank Sam MacDonald and André Phillion for their previous assistance during the X-ray tomography sessions at the Swiss Light Source. All electron microscopy measurements were obtained at the Centre Interdisciplinaire de Microscopie Electronique (CIME) at Ecole Polytechnique Fédérale de Lausanne (EPFL).

\section{References}

[1] Liu S, Napolitano R E and Trivedi R 2001 Acta Materialia 49 4271-4276

[2] Gonzales F and Rappaz M 2006 Metallurgical and Materials Transactions A: Physical Metallurgy and Materials Science 37 2797-2806

[3] Gonzales F and Rappaz M 2008 Metallurgical and Materials Transactions A: Physical Metallurgy and Materials Science 39 2148-2160

[4] Haxhimali T, Karma A, Gonzales F and Rappaz M 2006 Nature Materials 5 660-664

[5] Mullis A, Dragnevski K and Cochrane R 2004 Materials Science and Engineering A 375-377 157-162

[6] Assadi H, Oghabi M and Herlach D 2009 Acta Materialia 57 1639-1647

[7] Gonzales F 2009 Etude des morphologies de solidification dans les alliages aluminium-zinc Ph.D. thesis Ecole Polytechnique Fdrale de Lausanne

[8] Terzi S, Taylor J, Cho Y, Salvo L, Suéry M, Boller E and Dahle A 2010 Acta Materialia 58 5370-5380

[9] Limodin N, Salvo L, Boller E, Suéry M, Felberbaum M, Gailliègue S and Madi K 2009 Acta Materialia 57 $2300-2310$ 
[10] Ludwig O, Dimichiel M, Salvo L, Suéry M and Falus P 2005 Metall Mater Trans 361515

[11] Pompe O and Rattenmayr M 1998 Journal of Crystal Growth 192 300-306

[12] Stampanoni M, Groso A, Isenegger A, Mikuljan G, Chen Q, Bertrand A, Henein S, Betemps R, Frommherz U, Bhler P, Meister D, Lange M and Abela R 2006 Progress in Biomedical Optics and Imaging - Proceedings of SPIE 6318 1605-7422

[13] Friedli J, Fife J, Di Napoli P and Rappaz M 2012 Acta Materialia, Submitted

[14] Friedli J, Di Napoli P, Rappaz M and Dantzig J 2012 Proceedings of MCWASP 2012 ed Ludwig A

[15] Fife J L, Rappaz M, Pistone M, Celcer T, Mikuljan G and Stampanoni M 2012 Journal of Synchrotron Radiation, Accepted 